\title{
EFFICIENT ROTATING FRAME SIMULATION IN TURBOMACHINERY
}

\author{
L. Remaki, A. Ramezani \\ BCAM \\ Basque Center for Applied Mathematics \\ Bilbao, Spain \\ Email: Iremaki@bcamath.org \\ aramezani@bcamath.org
}

\author{
J.M. Blanco* \\ UPV/EHU \\ University of the Basque Country \\ Bilbao, Spain \\ Email: jesusmaria.blanco@ehu.es
}

\author{
J.I. Antolin \\ BALTOGAR \\ High Performance Industrial Fans \\ Bilbao, Spain \\ Email: jas@baltogar.com
}

\begin{abstract}
This paper deals with the simulation of steady flows in turbomachinery. Two approaches are proposed, the first one is the classical multiple-rotating frame method (MRF) by multizone approach where the different zones are separated by nonoverlapping interfaces and solved independently. Since each zone is loaded separately, a transferring system should be properly implemented at the interface boundaries. Two techniques are considered, in the first one the conservative variables are interpolated between zones while in the second one the fluxes are transferred through the interfaces.

The other proposed approach is a new version of the MRF using a virtual interface (VMRF). This is a simplified of the previous one where the interfaces are created virtually at the solver level, rendering the method easy to implement especially for edge-based numerical schemes, and avoiding any re-meshing in case one needs to change interface position, shape or simply remove or add new one. Finally, numerical tests are performed to demonstrate the efficiency of the proposed methods by comparison with commercial codes (ANSYS FLUENT).
\end{abstract}

\section{INTRODUCTION}

Accurate and cost effective simulation of vortex flow caused by fixed and rotating frames interaction is one of the major problems widely studied in turbomachinery. Such machines are extensively used in many industries including aerospace $[1,2]$, au- tomotive [3], ventilation [4], power generation, chemical manufacturing [5], petroleum exploration [6] and others. This demonstrates the importance of turbomachines optimal design which should be based on a realistic flow simulation. Many techniques to simulate rotating effect have been developed in the literature including the single rotating frame method, multiple rotating frame method, sliding surface method and so on. The multiple rotating frame (MRF) technique [7] is one of the mostly used for steady flows simulation. The technique consists of creating an interface between rotating and static parts of the geometry, then solve the Navier-Stokes equations in a relative frame (associated to the rotating part) and in a stationary frame. Information is transferred through the interface. Such a method is not easy to implement; first of all you need to create the interface at the CAD level which is not always an easy task, in particular for strongly connected rotating and static parts. Then we need to use a mesher capable to mesh more than one sub-domain.

Apart from geometrical manipulation, the interface has no physical existence but created for a numerical simulation considerations. Two different set of equations (in stationary and rotating frame) are solved at both sides of the interface, so its position will affect the results and convergence [8]. The optimal position is unfortunately not trivial and could not be accurately defined a before running the simulation. So we may need to adjust the position of the interface at the CAD level and remesh the domain many times. Finally a suitable boundary condition at the interface should be imposed. To simplify this method, we propose in this paper a simplified approach, we refer to it by VMRF

\footnotetext{
*ASME Member.
} 
(virtual multiple rotating frame), where the interfaces are created virtually at the solver level rendering the method easier to implement, especially for edge-based numerical schemes. Both methods VMRF and the classical MRF (multizones_MRF, using a multizone approach) are implemented in BBIPED (BcamBaltogar industrial platform for engineering design), a CFD platform being developed using open source SALOME (grid generator) [9] and SU2 (CFD solver) [10] as basis. Numerical tests are performed and results of both methods and commercial codes are compared.

\section{GOVERNING EQUATIONS}

The averaged equations governing three dimensional compressible inviscid flow are formulated relative to a Cartesian $\left(x_{1}, x_{2}, x_{3}\right)$ coordinate system, over a fixed volume $\mathscr{V}$ with a closed surface $\mathscr{S}$, in the integral form.

$$
\frac{\partial}{\partial t} \int_{\mathscr{V}} Q \mathrm{~d} \mathscr{V}=\int_{\mathscr{S}} F^{\alpha}(Q) n_{\alpha} \mathrm{d} \mathscr{S} \quad \text { for } \alpha=1,2,3
$$

where the summation convention is employed and $n=$ $\left(n_{1}, n_{2}, n_{3}\right)$ denotes the unit outward normal vector to $\mathscr{S}$. In this equation, the conservative variables $Q$ and the inviscid flux vectors $F^{\alpha}$ are defined respectively by:

$$
Q=\left(\begin{array}{c}
\rho \\
\rho u_{1} \\
\rho u_{2} \\
\rho u_{3} \\
\rho E
\end{array}\right) \quad F^{\alpha}=\left(\begin{array}{c}
\rho u_{\alpha} \\
\rho u_{1} u_{\alpha}+p \delta_{\alpha_{1}} \\
\rho u_{2} u_{\alpha}+p \delta_{\alpha_{2}} \\
\rho u_{2} u_{\alpha}+p \delta_{\alpha_{3}} \\
(\rho E+p) u_{\alpha}
\end{array}\right)
$$

where $\rho$ and $p$ denote the averaged density and pressure of the fluid respectively and $E=e+\frac{1}{2}\left(u_{\alpha} u_{\alpha}\right)$ is the total energy per unit mass, with $e$ being the internal energy per unit mass for the fluid, $u_{\alpha}$ is the averaged velocity of the fluid in direction $x_{\alpha}$ and $\delta_{\alpha i}$ is the Kronecker delta.

The equation set is closed by the addition of the ideal gas equation of state (i.e. $p=\rho(\gamma-1) e$, where $\rho$ is the density and $\gamma$ is the ratio of specific heats). Steady state solutions of the resulting equation set are defined in a fixed spatial computational domain $\Omega$.

\section{NUMERICAL DISCRETISATION}

On the generated consistent hybrid primal mesh, nodes are located at the vertices of the elements and the spatial discretisation of equation (1) is accomplished by using a cell vertex finite volume method [11]. This requires the construction of a dual mesh, in which each cell is associated with a single node of the primal mesh. For those regions in which the primal mesh consists only of isotropic tetrahedral cells, a median dual mesh is built by connecting cell edge midpoints, cell centroids and cell face centroids, such that only one node is present within each dual mesh cell [12-14]. With this strategy, each node $I$ of the domain mesh is associated with a volume $\Omega_{I}$ of the dual mesh. The boundary surface of such volume is denoted by $\Gamma_{I}$. Each edge of the domain mesh is associated with a segment of the dual mesh interface between the nodes connected to the edge. This segment is a surface built from triangular facets, where each facet is connected to the midpoint of the edge, a neighboring element centroid and the centroid of an element face connected to the edge, as illustrated in Fig. $1(a)$. The midpoint of the edge between node $I$ and $J$ is denoted by $x_{m}^{I J}$, the centroid of the face with vertices $I, J$ and $K$ is denoted by $x_{s}^{I J K}$ and the element centroid is designated by $x_{c}$. The bold lines on the dual mesh in this figure illustrate the boundaries between the edges in which the dual mesh segment is associated with. For this dual mesh definition, the volume $\Omega_{I}$ can be viewed as being constructed in terms of a set of tetrahedra, as illustrated for a typical interior node $I$ in Fig. $1(b)$. The surface of the dual mesh cell surrounding node $I$ is defined in terms of the closed set of planar triangular facets $\Gamma_{I}^{K}$, where each facet only touches a single edge of the domain mesh. The set of facets matching the edge between nodes $I$ and $J$ is denoted by $\Gamma_{I J}$.

In general, the median dual approach cannot be used for the hybrid elements produced by merging the stretched tetrahedra generated by the advancing layers method. This is because cells created in this way may be warped so severely that a vertex can lie outside the corresponding median dual cell. This may occur in regions of high curvature or at the interface between the hybrid and isotropic meshes. To overcome this problem, the information contained in the primal tetrahedral mesh is used to ensure that topology of the control volume cells is valid $[12,15]$. Equation (1) is applied to each cell $\Omega_{I}$ of the dual mesh. To perform the numerical integration of the inviscid fluxes over the surface $\Gamma_{I}$ of this cell, a set of coefficients is calculated for each edge using the dual mesh segment associated to the edge. The values of these coefficients for an internal edge are evaluated as $[13,14]$

$$
A_{I J}^{\alpha}=\sum_{K \in \Gamma_{I J}} A_{\Gamma_{I}^{K}} n_{\Gamma_{I}^{K}}^{\alpha}
$$

where $A_{\Gamma_{I}^{K}}$ is the area vector of facet $\Gamma_{I}^{K}$ and $n_{\Gamma_{I}^{K}}^{\alpha}$ is the component, in direction $x_{\alpha}$, of the outward unit normal vector of the facet from the viewpoint of node $I$. The integral of the inviscid flux over the surface $\Gamma_{I}$ is then approximated, using the summa- 


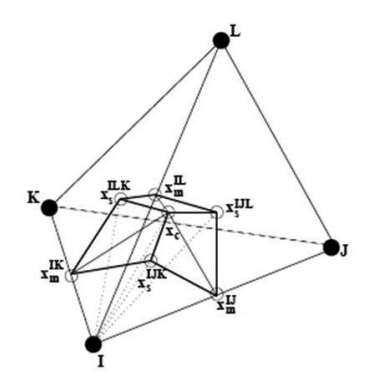

(a)

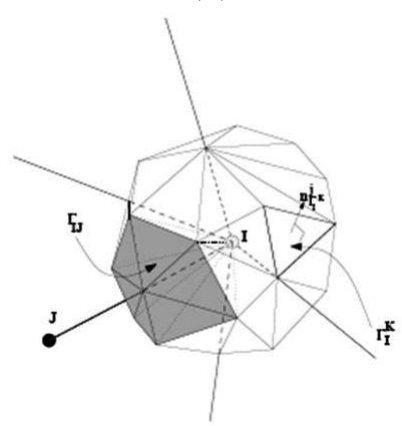

(b)

FIGURE 1. (A) ILLUSTRATION OF THAT PART OF THE DUAL MESH CELL SURROUNDING NODE $I$ WITHIN A TETRAHEDRAL CELL. (B) ILLUSTRATION OF THE DUAL MESH CELL SURROUNDING AN INTERNAL NODE $I$.

tion of edge contributions, as [14]

$$
\int_{\Gamma_{I}} F^{\alpha} d \Gamma \approx \sum_{J \in \Lambda_{I}} \tilde{F}_{I J} A_{I J}^{\alpha}
$$

where $\Lambda_{I}$ denotes the set of nodes connected to node $I$ by an edge in the domain mesh. Here,

$$
\tilde{F}_{I J}=\left(\begin{array}{c}
\rho q_{I J} \\
\rho u_{1} q_{I J}+p n_{I J}^{1} \\
\rho u_{2} q_{I J}+p n_{I J}^{2} \\
\rho u_{3} q_{I J}+p n_{I J}^{3} \\
(\rho E+p) q_{I J}
\end{array}\right)
$$

is a consistent numerical inviscid flux function and

$$
q_{I J}=n_{I J}^{\alpha}\left(u_{\alpha}\right)_{I}
$$

is the velocity in the direction of the edge connecting nodes $I$ and $J$. The solution is advanced in time to steady state using an explicit multi-stage Runge Kutta procedure and an implicit time stepping with biconjugate gradient stabilized solver. The convergence is accelerated by the use of local time stepping and by the addition of an agglomerated multigrid process.

\section{MULTI-ZONES APPROACH FOR MULTIPLE ROTATING FRAME METHOD (MRF)}

One of the most popular methods used to simulate the rotating effect is the so called Multiple Rotating Frame (MRF) introduced by $[7,10]$. Actually the flow for such problem is unsteady due to the existence of fixed (volute) and rotating parts (rotor) simultaneously in the domain. The MRF technique however, by putting the observer on the rotating axis, allows to view the problem as steady. The observer of the rotating domain will feel a steady state so any unsteadiness in boundaries violates the validity of the MRF assumption [16]. Therefore the definition of the interface which is the outer boundary of the rotating part is very important and misplacing it may cause divergence or non physical phenomena [17]. This approach consists of defining an axisymmetric sub-domain containing the rotating part endowed with a relative frame. The Euler equations are expressed in the relative frame to simulate rotating parts (in which they are considered static), while a stationary frame is used for the rest of the domain. A steady transfer of information is made through the interface separating the two domains. The Euler equations in the rotating frame are given by:

$$
\frac{\partial}{\partial t} \int_{\mathscr{V}} Q^{r} \mathrm{~d} \mathscr{V}=\int_{\mathscr{S}} F^{\alpha}\left(Q^{r}\right) n_{\alpha} \mathrm{d} \mathscr{S}+\int_{\mathscr{V}} S \mathrm{~d} \mathscr{V} \quad \text { for } \alpha=1,2,3
$$

$$
S=\left(\begin{array}{c}
0 \\
-2 \varepsilon_{i j}^{1} \omega_{i} u_{j}^{r}-\varepsilon_{k l}^{1} \varepsilon_{i j}^{k} \omega_{i} x_{j} \omega_{l} \\
-2 \varepsilon_{i j}^{2} \omega_{i} u_{j}^{r}-\varepsilon_{k l}^{2} \varepsilon_{i j}^{k} \omega_{i} x_{j} \omega_{l} \\
-2 \varepsilon_{i j}^{3} \omega_{i} u_{j}^{r}-\varepsilon_{k l}^{3} \varepsilon_{i j}^{k} \omega_{i} x_{j} \omega_{l} \\
0
\end{array}\right) \quad Q^{r}=\left(\begin{array}{c}
\rho \\
\rho u_{1}^{r} \\
\rho u_{2}^{r} \\
\rho u_{3}^{r} \\
\rho E^{r}
\end{array}\right)
$$

$$
u_{\alpha}^{r}=u_{\alpha}-\varepsilon_{i j}^{\alpha} \omega_{i} x_{j} \quad E^{r}=e+\frac{1}{2}\left(u_{\alpha}^{r} u_{\alpha}^{r}-\varepsilon_{i j}^{\alpha} \varepsilon_{k l}^{\alpha} \omega_{i} x_{j} \omega_{k} x_{l}\right)
$$

where $\varepsilon_{j k}^{i}$ is Levi-Civita symbol and $r$ superscript refers to the relative frame. $\omega$ is the angular velocity vector and $x$ is the Cartesian position vector of the cell, $P_{r} e, U_{r}$ denote the averaged density, pressure, total energy and velocity of the fluid in 


\section{REFERENCES}

[1] Ming Z., Yihua C., "Numerical Simulation of Rotor Flow Field Based on Overset Grids and Several Spatial and Temporal Discretization Schemes", Chinese Journal of Aeronautics, Volume 25, Issue 2, Pages 155-163, 2012.

[2] Adamczyk J.J., "Numerical Simulation of Multi-Stage Turbomachinery Flows", RTO AVT Symposium On Design Principles and Methods for Aircraft Gas Turbine Engines, 1998.

[3] Olander M., "CFD Simulation of the Volvo Cars Slotted Walls Wind Tunnel", Master?s Thesis in Solid and Fluid Mechanics, Department of Applied Mechanics, Chalmers University of Technology, 2011.

[4] Califano A., Steen S., "Analysis of different propeller ventilation mechanisms by means of RANS simulations", First International Symposium on Marine Propulsors, Trondheim, Norway, 2009.

[5] Singh K.K., Mahajani S.M., Shenoy K.T., Patwardhan A.W., Ghosh S.K., "CFD modeling of pilot-scale pumpmixer: Single-phase head and power characteristics", Chemical Engineering Science, Vol. 22, Issue 5, pp. 13081322, 2007.

[6] Wadnerkar D., Utikar R. P., Tade M. O., Pareek V. K., “CFD simulation of solid?liquid stirred tanks", Advanced Powder Technology, Volume 23, Issue 4, Pages 445-453, 2012.

[7] Luo J.Y., Gosman A.D and Issa I.R, "Prediction of impeller-induced flows in mixing vessels using multiple frames of reference", Inst. Chem. Eng. Sympo. Ser., Vol. 136, 549-556, 1994.

[8] Zadravec M., Basic S., Hribersek M., "The influence of rotating domain size in a rotating frame of reference approach for simulation of rotating impeller in a mixing vessel", Journal of Engineering Science and Technology, Vol. 2, No. 2, pp. 126 - 138, 2007.

[9] SALOME, The Open Source Integration Platform for $\mathrm{Nu}-$ merical Simulation,http://www.salome-platform.org, since 2005.

[10] Palacios F., Colonno M. R., Aranake A. C., Campos A., Copeland S. R., Economon T. D., Lonkar A. K., Lukaczyk T. W., Taylor T. W. R., and Alonso J. J., "Stanford University Unstructured (SU2): An open-source integrated computational environment for multi-physics simulation and design”, AIAA Paper 2013-0287, 51st AIAA Aerospace Sciences Meeting and Exhibit. Grapevine, Texas, USA, January 7th - 10th, 2013.

[11] Eymard R., Gallu T., Herbin R., Finite volume methods. Handbook of Numerical Analysis, North Holland, Amsterdam, 7:713-1020, 2000.

[12] Sørensen K. A., "A multigrid accelerated procedure for the solution of compressible fluid flows on unstructured hybrid meshes", PhD Thesis, University of Wales, Swansea, 2002.

[13] Barth T.J., "Aspect of unstructured grids and finite-volume solvers for the Euler and Navier-Stokes equations", In Lecture Notes Presented at the VKI Lecture Series, Rhode Saint Genese Begium, 2 1995. Von karman Institute for fluid dynamics, 1994-05.

[14] Blazek J., Computational Fluid Dynamics: Principles and Applications, Elsevier, 2001.

[15] Sørensen K. A., Hassan O., Morgan K., Weatherill N. P., "A multigrid accelerated hybrid unstructured mesh method for 3D compressible turbulent flow", Comp.Mech. 31 (2003), 101-114.

[16] Denton J.D., "Some limitations of turbomachinery CFD", ASME Paper GT2010-22540,2010.

[17] Liu Z., Hill D.L., "Issues Surrounding Multiple Frames of Reference Models for Turbo Compressor Applications", International Compressor Engineering Conference,Paper 1369, 2000.

[18] Ansys Fluent, 2005, http://www.ansys.com/Products/ Simulation+Technology/Fluid+Dynamics/Fluid+Dynamics +Products/ANSYS+Fluent 